\section{PROCEEDINGS A}

royalsocietypublishing.org/journal/rspa

\section{Research}

Cite this article: Sircar A, Patra PK, Batra RC. 2020 Casimir force and its effects on pull-in instability modelled using molecular dynamics simulations. Proc. R. Soc. A 476: 20200311.

http://dx.doi.org/10.1098/rspa.2020.0311

Received: 22 April 2020

Accepted: 28 September 2020

\section{Subject Areas:}

computational physics, computer modelling and simulation

\section{Keywords:}

Casimir forces, pull in instability, molecular dynamics simulations, carbon nanotubes

\section{Author for correspondence:}

Puneet Kumar Patra

e-mail: puneet.patra@civil.iitkgp.ac.in

\section{Casimir force and its effects on} pull-in instability modelled using molecular dynamics simulations

\section{Avirup Sircar ${ }^{1}$, Puneet Kumar Patra ${ }^{2}$ and}

\section{Romesh (Batra ${ }^{3}$}

${ }^{1}$ Department of Mechanical Engineering, Indian Institute of

Technology Kharagpur, West Bengal 721302, India

${ }^{2}$ Department of Civil Engineering and Centre for Theoretical Studies, Indian Institute of Technology Kharagpur, West Bengal 721302, India

${ }^{3}$ Biomedical Engineering and Mechanics, M/C 0219, Virginia

Polytechnic Institute and State University, Blacksburg,

VA 24061, USA

PKP, 0000-0002-8585-8684

We present a new methodology to incorporate the Casimir forces within the molecular dynamics (MD) framework. At atomistic scales, the potential energy between two particles arising due to the Casimir effect can be represented as $U\left(r_{i j}\right)=C / r^{7}$. Incorporating the Casimir effect in MD simulations requires the knowledge of $C$, a problem hitherto unsolved. We overcome this by equating the total potential energy contributions due to each atomistic pair with the potential energy of continuum scale interacting bodies having similar geometries. After having identified the functional form of $C$, standard MD simulations are augmented with the potential energy contribution due to pairwise Casimir interactions. The developed framework is used to study effects of the Casimir force on the pull-in instability of rectangular and hollow cylindrical shaped deformable electrodes separated by a small distance from a fixed substrate electrode. Our MD results for pull-instability qualitatively agree with the previously reported analytical results but are quantitatively different. The effect of using longerranged Casimir forces in a constant temperature environment on the pull-in behaviour has also been studied. 


\section{Introduction}

In 1948, Casimir predicted that two perfectly conducting parallel metal plates separated by a small distance modified the ground state energy of the electromagnetic field to induce an attractive force between the plates [1]. In general, these forces manifest between any two uncharged bodies separated by a few nanometres $(\mathrm{nm})$ to micrometres $(\mu \mathrm{m})$. This discovery, now termed as the Casimir effect, has gained significant attention over the last few decades from researchers working on micro- and nano-devices. For simplicity, researchers have often treated the Casimir forces similar to the van der Waals forces. That is, the Casimir force is taken as the long-ranged version of the traditional van der Waals force. Both these forces originate from the electrostatic interaction among dipoles [2]. It is found that at separations of the order of a few tens of nanometres, where the effect of the finite speed of light becomes important [3], the van der Waals forces give way to the Casimir forces. It is important to note that both forces describe the same physical phenomenon and are related through the ground state energy oscillations of the electromagnetic field [4-6] with the main difference being their range of applicability [7]. Consequently, they do not act simultaneously on any system - at shorter separation only van der Waals forces act while at larger separation only Casimir forces prevail. Analytical expressions of the two forces for a simple geometry like parallel infinite plates differ marginally-Casimir (van der Waals) force is inversely proportional to the fourth (third) power of the separation distance [7]. The difference in expressions can be understood reasonably well by summing up the pairwise interactions of the atoms of two bodies that vary as $r^{-6}$ for the van der Waals forces and as $r^{-7}$ for the Casimir forces. The transition from the van der Waals to the Casimir forces occurs smoothly over a range of separation distance. Experimental investigations have demonstrated this transition to occur between 10 and $100 \mathrm{~nm}$ for a sphere-plate geometry in mica [8].

Research into the Casimir effect has transcended from being theoretical to experimental with the advancement in technology. While still a majority of research efforts focus on developing analytical expressions of Casimir forces for bodies of different geometry-parallel plates [1], rectilinear piston [9], plate-sphere [10-12], plate-cylinder [13,14], interesting experiments have been devised to accurately measure the force for different configurations [4,5,15]. Special focus has been placed on the analytical expressions of the Casimir interactions for geometries like plate-cylinder and plate-sphere because of their relevance in micro-electro-mechanical systems (MEMS) and nano-electro-mechanical systems (NEMS). The presence of Casimir forces in MEMS and NEMS devices poses formidable challenges during fabrication and operation phases [7]. As these devices consist of moving parts that are typically separated by a few micro/nanometres, Casimir interactions along with the Coulomb force can cause the moving parts to collapse on to each other. With increasing applications of electrically activated MEMS and NEMS as transistors, switches, micro-mirrors, pressure sensors, pumps, moving valves and shuttle memory devices [16-20], it is important to understand the effects of Casimir forces from molecular dynamics (MD) simulations.

A typical MEMS and NEMS device comprises of a conductive material separated by a small distance from a rigid and stationary substrate [21,22]. Under the application of an external potential, the deformable body deflects, changing the capacitance of the device. This principle has been used to develop devices for mass and chemical sensing [23-25]. The complex interplay among the elastic restoring, the attractive Coulomb and the Casimir forces determine the temporal evolution of the deformable body. If the net attractive force overcomes the elastic restoring force, the deformable body touches the substrate, and the functionality of the device is lost. This phenomenon, termed pull-in instability, has been observed experimentally $[26,27]$. The critical potential difference that causes pull-in instability is called the pull-in voltage, and the corresponding displacement of the device the pull-in displacement. The accurate determination of these two pull-in parameters enables designers of MEMS and NEMS devices to increase their performance [18] and avoid instabilities that prevent stable dynamics [28,29].

Most of the research concerning the effects of Casimir forces on MEMS and NEMS devices has revolved around developing analytical models of the fixed and the deformable electrodes 
using continuum beam and plate theories. The deformable electrode in the form of a flat body of thickness much smaller than its other dimensions has been analysed as a plate using both linear and nonlinear theories [30-37]. Electrodes having in-plane stiffness much higher than the bending stiffness have been studied as either linear or nonlinear elastic membranes [21,22,38-40]. For deformable electrodes comprised of rectangular members like nano-beams, different beam theories [41-47] have been adopted. Researchers have tried to understand the pull-in instability by adding contributions of the integrated form of molecular scale forces to the continuum models. For deformable electrodes having a separation gap usually smaller than $20 \mathrm{~nm}$, the van der Waals force, $F_{\mathrm{vdw}}$, given by $[41,48]$

$$
F_{\mathrm{vdw}}=\frac{A_{H} w L}{6 \pi(g-y)^{3}}
$$

is taken along with the electrostatic interaction (the Coulomb force) as the external forces. For separation gap generally larger than $20 \mathrm{~nm}$, the Casimir force, $F_{\text {cas }}$ given by [41]

$$
F_{\text {cas }}=\frac{\pi^{2} h c w L}{240(g-y)^{4}}
$$

replaces $F_{\mathrm{vdw}}$. Here, $A_{H}$ represents the Hamaker's constant, $w$ the width, $L$ the length, $g$ the initial separation distance between the bottom of the deformable electrode and top of the rigid grounded substrate, $y$ the vertically downward displacement of the deformable electrode, $h$ the reduced Planck's constant and $c$ the speed of the light.

The pull-in instability problem posed in this manner results in a distributed nonlinear system [49] which can be either analytically or numerically analysed. Several reduced order models (ROMs) have been proposed-some accounting for only van der Waals forces and others for only Casimir forces. The first such effort by Nathanson et al. [27] involved modelling a transistor as a lumped spring-mass system wherein the elastic restoring force per unit displacement is represented by the spring stiffness. The resulting pull-in voltage and displacement did not agree well with experimental observations [50] as this model does not capture nonlinearities due to the electrostatic interactions and the molecular scale forces [51]. Pamidighantam et al. [50] refined this model by considering a nonlinear spring that improved the estimate of the pull-in voltage but required an empirical value of the pull-in displacement. In order to obtain better agreement with experimental observations, researchers have used a nonlinear beam equation to predict the pullin parameters [23,52]. If the mode shape corresponding to the fundamental frequency is taken as the basis function, then the one-degree of freedom ROM predicts pull-in parameters close to those given by solutions of three-dimensional equations by the finite element method [53]. Serry et al. [54] have developed a ROM to study the effect of Casimir forces by assuming that the deformable electrode behaves as a rectangular membrane, while Lin \& Zhao [55] obtained analytical expressions of the critical pull-in parameters by using a perturbation theory. ROMs for rectangular and circular plates incorporating Casimir forces are described in [56,57]. Recently, non-local beam theories have been employed [41] to study the pull-in parameters.

With continuous miniaturization of devices, a natural question arises concerning the applicability of classical continuum scale governing equations to capture size effects observed at small-scales [58]. As a result, atomistic tools like MD simulations have been used to obtain fundamental insights into the pull-in phenomenon [59-61]. These MD results in the elastic regime agree well with those from the continuum models. Researchers have enriched the MD simulations by considering different defects present in the nanotubes [60]. However, to the best of our knowledge, MD simulations performed thus far have considered only the van der Waals force and not the Casimir force. We conjecture that it may be because of the difficulty in finding the appropriate expression for the Casimir potential at the molecular level.

Here, we propose a framework for incorporating Casimir interactions in MD simulations. The developed framework is used to assess the effect of Casimir forces on the mechanical behaviour and the pull-in instability. Although, the Casimir potential involves contributions beyond the pairwise interactions, the present work is limited to pairwise interactions. The potential functional is obtained by equating the summed up pair-wise interactions with the analytical expression 
of the continuum scale Casimir energy. Furthermore, we assume that the system is present in vacuum so that effects of the dielectric medium do not enter into the analysis. The resulting potential is applied to two different electrode geometries-(i) an NEMS device whose deformable electrode is a Silicon nano-wire of rectangular cross section, and (ii) an NEMS device having a cantilever carbon nanotube (CNT) as the electrode. For both cases, a single-layered graphene sheet is taken as the substrate. The computed MD results qualitatively agree with those deduced from a continuum theory. The effects of the thermal environment and the nature of the Casimir potential on the dynamical behaviour are investigated.

The rest of the manuscript is organized as follows. Section 2 details challenges encountered in incorporating the Casimir effect in the MD simulations, and how they are addressed. Section 3 provides details of the NEMS geometries and the computational work. In §4, we quantity and compare results of MD simulations with different theoretical formulations. Conclusions and the path forward are given in $\S 5$.

\section{Incorporating Casimir effect in molecular dynamics simulations}

\section{(a) Inter-particle Casimir interaction}

The pairwise Casimir potential between two particles $A$ and $B$, separated by a distance $r$, is given by [62]:

$$
U_{\text {cas,pair }}(r)=-\frac{\hbar c}{4 \pi r^{7}}\left[23\left(\alpha_{E}^{A} \alpha_{E}^{B}+\alpha_{M}^{A} \alpha_{M}^{B}\right)-7\left(\alpha_{E}^{A} \alpha_{M}^{B}+\alpha_{M}^{A} \alpha_{E}^{B}\right)\right],
$$

where, $h$ is the reduced Planck's constant, $c$ is the speed of light, and $\alpha_{E}$ and $\alpha_{M}$ are the electric and magnetic polarizability of the materials. For simplicity, we consider two materials that are purely electrically polarizable so that $\alpha_{M}=0$. Thus equation (2.1) simplifies to [63]

$$
U_{\text {cas,pair }}(r)=\frac{-23 \hbar c \alpha_{E}^{A} \alpha_{E}^{B}}{4 \pi r^{7}}=-\frac{C}{r^{7}} .
$$

The inter-particle interaction shown in equation (2.2) results in a finite macroscopic expression of the Casimir energy that can be obtained by performing an integration over all particle pairs of the two bodies.

\section{(b) Macroscopic Casimir energy from inter-particle interaction}

The two interacting surfaces initially separated by gap $g$ for our case and shown in figure 1 correspond to-(i) a thin cylinder and a plate, and (ii) a solid rectangular beam and a plate. To calculate the total Casimir energy, it is assumed that each particle pair interacts through equation (2.2). Let $n_{A}$ and $n_{B}$ represent the number densities of the particles, where the cylindrical/rectangular body is represented by subscript $A$ and the infinite plate is represented by subscript $B$. In order to derive the total Casimir energy, we first begin with the Casimir energy between a point particle and a plate separated by distance $g$. In a ring of radius $d x$ at a horizontal distance $x$ from the particle, the total charge present on the plate is: $n_{B} \times 2 \pi x d x$. Therefore, the total Casimir energy $U$ between the point particle and the infinite plate is given by

$$
U=\int_{0}^{\infty} \frac{C\left(n_{B} 2 \pi x \mathrm{~d} x\right)}{\left(g^{2}+x^{2}\right)^{7 / 2}}=\int_{g}^{\infty} \frac{C\left(n_{B} 2 \pi t \mathrm{~d} t\right)}{t^{7}}=\frac{2 \pi n_{B} C}{5 g^{5}} .
$$

For the cylinder-plate geometry, the total charge on the cylinder equals $2 \pi L R n_{A}$. Under the assumption, $R \ll g$ and $L \sim g$, the cylinder may be treated as a point mass (since the plate is infinite in two dimensions) with the total charge $2 \pi L R n_{A}$, so that the net Casimir energy equals $U \times 2 \pi L R n_{A}$. The steps for deriving the total Casimir energy for the rectangular-plate interaction with the substrate are the same. Consider a thin strip of the rectangular body of length $\mathrm{d} y$, located at a distance $y$ above the plate. This thin strip has a total charge of $n_{A} w L \mathrm{~d} y$, and is treated as a point mass. This strip, therefore, interacts with the plate through the energy: 

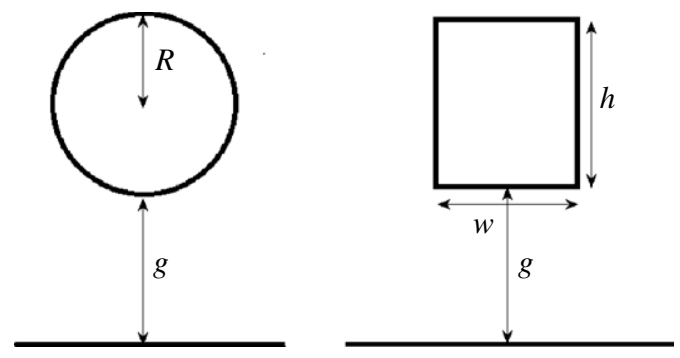

Figure 1. Cross sections of deformable and stationary electrodes initially separated by distance $g$ (sketch not to scale).

$\mathrm{d} U=2 \pi n_{B} C n_{A} w L \mathrm{~d} y / 5 y^{5}$. Integration over $\mathrm{d} y$ gives the total Casimir energy. The mathematical expressions for the Casimir energy in cylinder plate and rectangular body-plate geometries are as follows:

$$
\begin{aligned}
U_{\text {cas }} & =\frac{2 \pi^{2} C n_{A} n_{B}}{5} \times \frac{2 L R}{g^{5}}: \text { cylinder-plate } \\
& =\frac{\pi C n_{A} n_{B} w L}{10}\left(\frac{1}{g^{4}}-\frac{1}{(g+h)^{4}}\right): \text { rectangular-plate. }
\end{aligned}
$$

Assuming that the materials are conducting, and employing the Clausius-Mosotti relation [63], equation (2.4) can be further simplified to

$$
\begin{aligned}
U_{\text {cas }} & =\frac{207 \hbar c}{80 \pi} \frac{L R}{g^{5}}: \text { cylinder-plate } \\
& =\frac{207 \hbar c w L}{640 \pi^{2}}\left(\frac{1}{g^{4}}-\frac{1}{(g+h)^{4}}\right): \text { rectangular-plate }
\end{aligned}
$$

Equation (2.5) provides an estimate of the macroscopic Casimir energy occurring due to atomistic pair-wise interactions. However, the resulting macroscopic energies obtained from equation (2.5) considerably differ from those computed using the well established macroscopic expressions. Using the proximity force approximation (PFA), where it is assumed that the two interacting surfaces are smooth, almost parallel and close to each other so that the Casimir energy for two parallel plates may be used [64], the expression of Casimir energy for cylinder-plate geometry becomes [14]:

$$
U_{\mathrm{PFA}}=-\frac{\pi^{3} \hbar c L}{960} \sqrt{\frac{R}{2 g^{5}}} .
$$

Emig et al. $[13,14]$ have derived an expression for the Casimir energy without making the PFA. They found that for a plate-cylinder geometry, Casimir forces have one of the weakest possible decay, and the Casimir potential is given by $[13,14]$

$$
U_{\mathrm{LOG}}=-\frac{\hbar c L}{(g+R)^{2}} \frac{1}{16 \pi \log \left(\frac{g+R}{R}\right)} .
$$

For the plate-plate geometry, neglecting deflections, the macroscopic expression of the Casimir energy is (integrating the force given in equation (1.2) [55])

$$
U_{\operatorname{Rec}}=\frac{\pi^{2} \hbar c w L}{720 g^{3}} .
$$

It is clear that $U_{\text {cas }}$ obtained from equation (2.5) significantly differs from that obtained using equations (2.6), (2.7) and (2.8). This may be exemplified by considering a deformable electrode made of an armchair $(15,15)$ CNT of $R=1 \mathrm{~nm}$ and $L=100 \mathrm{~nm}$. Let the CNT be separated from a grounded electrode made of graphene by a distance $g=50 \mathrm{~nm}$. Here, $n_{A} \sim 73$ atoms $\mathrm{nm}^{-2}$, 
$n_{B} \sim 39$ atoms $\mathrm{nm}^{-2}$. Taking the reduced Planck's constant as $6.582 \times 10^{-16} \mathrm{eVs} \mathrm{rad}^{-1}$, and c as $3 \times 10^{17} \mathrm{~nm} \mathrm{~s}^{-1}$ and substituting these in equations (2.5), (2.6) and (2.7), we get

$$
\left.\begin{array}{l}
U_{\text {cas }}=5.204 \times 10^{-5}, \\
U_{\text {PFA }}=2.551 \times 10^{-2}, \\
U_{\text {LOG }}=8.845 \times 10^{-2} .
\end{array}\right\}
$$

Note the significant difference between the different estimates of the Casimir energy obtained using three different approximations. The energy obtained by integrating all pairwise contributions is off by three orders of magnitude. The discrepancy further increases as one drops the Clausius-Mosotti equation and uses the polarizability values directly obtained from quantum mechanical calculations. The discrepancy may be attributed to the contribution to the Casimir energy from multi-body interactions-the interaction energy of two particles is altered by the presence of the third particle [63]. The multibody terms that account for the entire Casimir energy within the framework of MD simulations is yet to be found. To make matters further complicated, the polarizability of CNTs changes with their geometry [65]. We have not come across MD simulations of systems interacting via Casimir forces.

\section{(c) Finding a pair potential that provides a reasonable total energy}

We address the issue of mismatch between pair summation and total energy by treating $C$ as a distance dependent function. For a rectangular beam-plate interaction, we equate the Casimir energy given by equations (2.8) and (2.4) to obtain for $C$ the following expression:

$$
C_{\operatorname{Rec}}=\frac{\pi}{72} \times \frac{\hbar c}{n_{A} n_{B}} \times \frac{g}{1-\frac{g^{4}}{(g+h)^{4}}} .
$$

For a cylinder-plate geometry, a similar process gives the following two different expressions for $C$.

(i) Equations (2.4) and (2.6), yield

$$
C_{\mathrm{PFA}}=\frac{\pi}{768} \times \frac{\hbar c}{n_{A} n_{B}} \sqrt{\frac{g^{5}}{2 R}} .
$$

(ii) Similarly, equations (2.4) and (2.7) provide

$$
C_{\mathrm{LOG}}=\frac{5 h c}{64 \pi^{3} n_{A} n_{B}} \times \frac{g^{5}}{(g+R)^{2}} \times \frac{1}{\log \left(\frac{g+R}{R}\right)} .
$$

This method of finding the functional form of $C$ is empirical and is based on equating the macroscopic Casimir energy with its atomistic counterpart obtained by pair-wise summation of energies. However, interactions obtained through this approach may induce other non-physical effects in the system. Furthermore, this approach does not account for altered interaction due to load induced changes in geometry.

Upon obtaining the functional form of $C$ (see equations (2.10) (2.11) and (2.12)), the Casimir potential in pair-wise form between two particles is implemented through equation (1.2) within the MD framework. The contributions arising due to the Coulomb potential, interatomic interactions between the substrate particles and the electrode particles, and the van der Waals potential supplement the pair-wise Casimir potential. 


\section{System and simulation details}

We now briefly explain the system studied and the simulation details. The present work is limited to a cantilever NEMS device shown in figure 2 .

\section{(a) Geometry}

Three sets of simulations have been designed with two different geometries - (i) one where the deformable electrode is a silicon beam of rectangular cross section having dimensions $L=10 \mathrm{~nm}$, $w=6 \mathrm{~nm}$ and $h=1.5 \mathrm{~nm}$ [66], (ii) the second also has a silicon nano beam as an electrode but with a square cross section of dimensions $L=8 \mathrm{~nm}, w=4 \mathrm{~nm}$ and $h=4 \mathrm{~nm}$, and (iii) in the last, the deformable electrode is a cylindrical $(5,5) \mathrm{CNT}$ of length $L=10 \mathrm{~nm}$. In all cases, the rigid substrate is made of a single layer graphene sheet with $L_{X}=L_{Y}=10 \mathrm{~nm}$ for simulation sets (i) and (iii), and $L_{X}=8 \mathrm{~nm}$ and $L_{Y}=10 \mathrm{~nm}$ for simulation set (ii). The axes of the deformable electrodes are oriented symmetrically with respect to the substrate. Rigidity is provided at the fixed end, shown as the shaded region in figure 2. For simulation sets (i) and (ii), the length of this region equals $2 \mathrm{~nm}$, while in simulation set (iii) it equals $1 \mathrm{~nm}$. The initial separation distance, $g$, has been taken as a variable. Note that typically in NEMS both the electrode's length and the separation distance are larger than those used here. Because the forces involved in these simulations are long ranged, we take smaller dimensions to save computational time. As is often done when studying NEMS, the graphene sheet is kept stationary throughout the simulation. Note that a correction factor is usually used to model semiconductors like silicon instead of metals with Casimir forces [67]. However, for simplicity, we have not used it in this work.

\section{(b) Interaction potential}

The atoms of the electrodes and the substrate are subjected to three types of interaction potentials, as detailed below.

(i) Interactions within the electrode and the substrate: The interatomic interactions within the electrode as well as the substrate are modelled through three-body Tersoff potentials [68] -all Silicon-Silicon interactions have been modelled using the Tersoff potential for Silicon while all Carbon-Carbon interactions have been modelled using the Tersoff potential for Carbon. The Tersoff potential is given by the following equation:

$$
\begin{aligned}
& E=\sum_{i} E_{i}=\frac{1}{2} \sum_{i \neq j} \sum_{j} \phi\left(r_{i j}\right), \\
& \phi\left(r_{i j}\right)=f_{c}\left(r_{i j}\right)\left[f_{R}\left(r_{i j}\right)+b_{i j} f_{A}\left(r_{i j}\right)\right],
\end{aligned}
$$

where $E$ denotes the total potential energy of the system, $E_{i}$ the potential energy of the $i$ th atom, and $\phi$ the potential energy between the $i$ th and the $j$ th atoms. The other variables in equation (3.1) have the following meaning: $r_{i j}$ represents the distance between the $i$ th and the $j$ th atoms, $b_{i j}$ is the bond order function, $f_{C}$ the cut-off function that ensures nearestneighbour interaction, $f_{R}$ the repulsive pair potential, and $f_{A}$ the attractive pair potential. The mathematical forms of these functions are given below

$$
\begin{aligned}
& f_{c}\left(r_{i j}\right)= \begin{cases}1 & \forall r_{i j} \leq P_{i j} \\
\frac{1}{2}-\frac{1}{2} \sin \left(\frac{\pi}{2} \frac{r_{i j}-R_{i j}}{D_{i j}}\right) & \forall P_{i j}<r_{i j}<Q_{i j} \\
0 & \forall r_{i j}>Q_{i j}\end{cases} \\
& f_{R}\left(r_{i j}\right)=A \mathrm{e}^{-\lambda_{1} r_{i j}}, f_{A}\left(r_{i j}\right)=-B \mathrm{e}^{-\lambda_{2} r_{i j}}, \\
& b_{i j}=\left(1+\beta^{n} \zeta_{i j}^{n}\right)^{-\frac{1}{2 n}},
\end{aligned}
$$




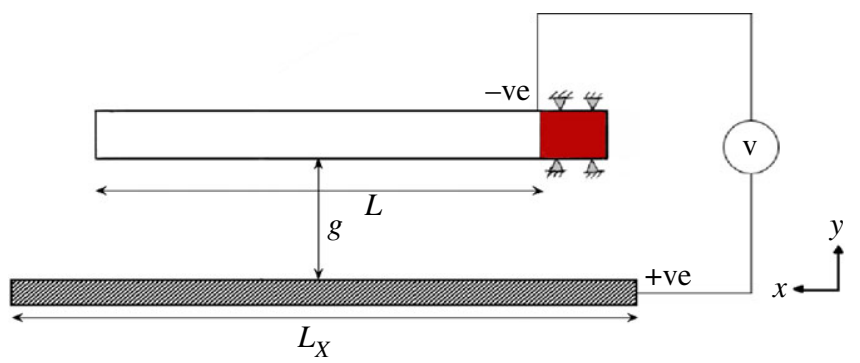

Figure 2. Schematic of the simulation model. A cantilever beam of rectangular/circular cross section makes the deformable electrode of length $L$. It is separated by a distance $g$ from the substrate made of a graphene sheet of dimensions: $L_{x} \times L_{\gamma}$. The substrate is fixed to the ground while the region shown in brown provides fixity to the nano-beam. (Online version in colour.)

$$
\begin{aligned}
& \zeta_{i j}=\sum_{k \neq i, j} f_{c}\left(r_{i k}\right) g\left(\theta_{i j k}\right) \exp \left[\lambda_{3}^{3}\left(r_{i j}-r_{i k}\right)^{3}\right], \\
& \left.g\left(\theta_{i j k}\right)=1+c^{2} / d^{2}-c^{2} /\left[d^{2}+\left(h-\cos \theta_{i j k}\right)^{2}\right)\right] .
\end{aligned}
$$

Here, $P_{i j}=R_{i j}-D_{i j}, Q_{i j}=R_{i j}+D_{i j}$. The cut-off function is continuous and goes from 1 to 0 smoothly as the distance varies from $P_{i j}$ to $Q_{i j}$. $R_{i j}$ is chosen to include only the firstneighbour shell for most problems of interest. $\theta_{i j k}$ is the angle between the bonds $i j$ and $i k$. Depending upon the system being simulated, the parameters in the equation take different values. In the present work, parameters proposed by Lindsay \& Broido [69] have been adopted for Carbon-Carbon interactions while those by Tersoff [68] for SiliconSilicon interactions. The potential parameters chosen here have been extensively used to obtain thermal and mechanical properties of different Carbon- and Silicon-based nanoscale systems [70-73].

(ii) Electrostatic potential between the electrode and the substrate: The interatomic interaction for studying pull-in instability is incomplete without an appropriate model to account for the electrostatic attraction between the electrode and the substrate. As a potential difference is created between them, charges of opposite sign are induced. Consequently, attractive electrostatic forces develop between the two electrodes. We model this electrostatic potential using pairwise Coulomb potential, $U_{\text {coul }, i j}$ :

$$
U_{\mathrm{coul}, i j}=\frac{q_{i} q_{j}}{r_{i j}}, r_{i j}<r_{\mathrm{coul}} \text {. }
$$

Here, $q_{i}$ and $q_{j}$, respectively, represent the charge on atoms $i$ and $j$ with $r_{i j}$ equalling the distance between them. The electrostatic attraction cuts off at a distance of $r_{\text {coul }}=$ 12,10 and $10 \mathrm{~nm}$ for simulation sets (i), (ii) and (iii) respectively.

(iii) Cross interactions between the electrode and the substrate: The cross interactions between the electrode and the substrate are modelled through short-ranged forces using the van der Waals and the Casimir interaction potentials. The Casimir potential between two atoms, as shown in equation (2.2) with $C$ given by equation (2.10) for simulation sets (i) and (ii), and by equations (2.11) and (2.12) for simulation set (iii), is considered for a separation distance greater than $1 \mathrm{~nm}$. As mentioned above, the cut-off distance for simulation set (i) is $12 \mathrm{~nm}$ while that for simulation sets (ii) and (iii) is $10 \mathrm{~nm}$. At a separation distance less than $1 \mathrm{~nm}$, the Casimir potential is replaced by the van der Waals potential. We model the van der Waals potential through the standard 6-12 Lennard-Jones potential:

$$
U_{\mathrm{vdw}, i j}=4 \epsilon\left[\left(\frac{\sigma}{r_{i j}}\right)^{12}-\left(\frac{\sigma}{r_{i j}}\right)^{6}\right] .
$$


Here, $\epsilon$ equals $0.00485 \mathrm{eV}$ while $\sigma$ equals $0.401 \mathrm{~nm}$ for set (iii). For sets (i) and (ii) the Lorentz-Berthelot mixing rule [71] has been used to obtain $\epsilon=0.00347 \mathrm{eV}$ and $\sigma=$ $0.376395 \mathrm{~nm}$. For set (iii) $\epsilon=0.00485 \mathrm{eV}$ and $\sigma=0.401 \mathrm{~nm}$. The van der Waals potential is cut off to zero beyond $r_{\mathrm{vdw}}=1 \mathrm{~nm}$.

It has been reported that for gold-coated aluminium surfaces [6], van der Waals forces are effective within a distance of $0.5-4 \mathrm{~nm}$. For the distance between $4 \mathrm{~nm}$ and $1 \mu \mathrm{m}$, a transition from the van der Waals to the Casimir forces occurs. For distances exceeding $1 \mu \mathrm{m}$, the Casimir forces act. Since our simulation geometry is rather small, we assumed that the transition from the van der Waals to the Casimir instantaneously occurs at a distance of $1 \mathrm{~nm}$. The intent here is to illustrate effects of this transition rather than model an actual device.

\section{(c) Simulation methodology}

The simulation begins with defining the geometry of the electrode and the substrate. Initially, the electric charge on each atom is taken as zero. Following a conjugate gradient based minimization, constant temperature (NVT) equilibration runs are performed for 100000 time steps of size $0.001 \mathrm{ps}$. Two separate constant temperature environments are considered for elucidating effects of the temperature on modelling Casimir forces-one at a temperature of $1 \mathrm{~K}$ and other at $300 \mathrm{~K}$. While finite temperature corrections to the Casimir potential need to be modelled separately, the present study excludes them. After equilibration, charges are assigned to both the electrode and the substrate atoms. The charge on each electrode atom is fixed at -1 electron charge, that on each substrate atom is incremented in a loop. Each such charge increment is followed by 200000 constant temperature MD simulation runs. Instead of using a deterministic thermostat [74-77], the stochastic Langevin thermostat is used for the NVT simulations. All simulations have been performed in the free-to-use software LAMMPS. The essential features of the dynamics are extracted by monitoring the trajectory of the bottommost particle at the free end for the cantilever device.

\section{Results}

We present the pull-in instability results in this section and the effects of incorporating the Casimir forces vis-á-vis the van der Waals forces alone. This section begins by comparing results obtained from our MD model with those obtained from continuum models [60,61]. It is important to note that it is the first attempt to model Casimir forces through MD simulations, and predictions from the continuum and the MD models may not be close to each other. Subsequently, effects of geometric parameters and the long-range Casimir forces are delineated by computing the pull-in charge.

\section{(a) Comparison with existing literature results for a rectangular cross section beam}

Since analytical expressions of the pull-in instability have invariably involved rectangular beamplate interactions, the comparison with existing literature results is made only for simulation sets (i) and (ii), i.e. for a rectangular beam-plate geometry. For simulation sets (i) and (ii) $g=5$ and $2.5 \mathrm{~nm}$ are used, respectively. Under the assumption that before the pull-in instability occurs, the electrode-substrate system behaves as a capacitor, the charge, $q_{\mathrm{PI}}$, at which the pull-in occurs is a proxy of $V_{\text {PI }}$ since $q \propto V$ for a capacitor. Separate simulations have been carried out to find the pull-in charge with only the van der Waals forces $\left(q_{\mathrm{PI}, \mathrm{vdW}}\right)$, and with a switch from the Casimir to the van der Waals forces $\left(q_{\mathrm{PI}, \mathrm{cas}-\mathrm{vdW}}\right)$. Throughout the remainder of this paper, we denote cases with only the van der Waals forces as only-vdW, while those with the switch from the Casimir to the van der Waals forces as Casimir-vdW. The pull-in charge, $q_{\mathrm{PI}}$, for each case is found and the ratio, $Q_{\mathrm{md}}=q_{\mathrm{PI}, \mathrm{only} \text {-vdW }} / q_{\mathrm{PI}, \text { casimir-vdW }}$, is compared with the ratio of the pull-in voltages obtained theoretically. 
(a)

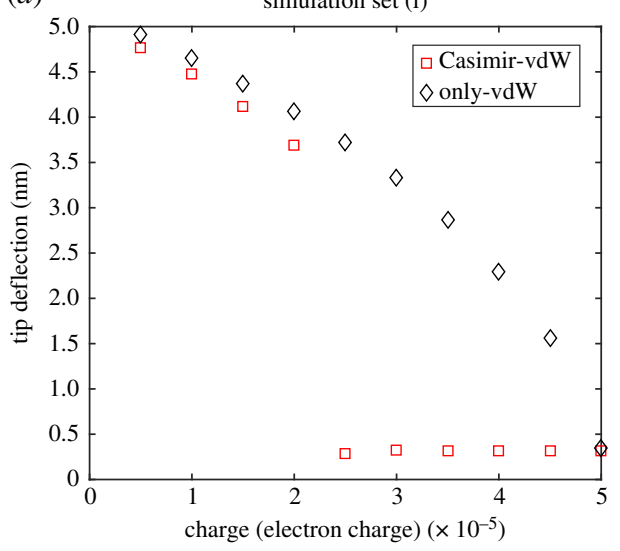

(b)

simulation set (ii)

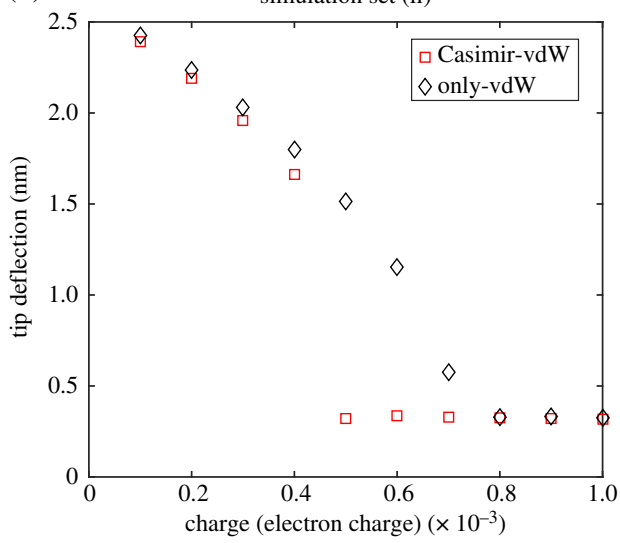

Figure 3. The free-end tip deflection with an increase in the charge for (a) simulation set (i) and (b) simulation set (ii). For both cases, the pull-in charge on each atom is more when only the van der Waals forces are considered. For the Casimir-vdW case, the pull-in charge is much smaller. For (b), the pull-in charge is an order of magnitude higher than that for (a) owing to the higher bending stiffness and the smaller length of the beam. (Online version in colour.)

The free end tip deflection for the two simulation sets are shown in figure 3 . The pull-in charge is significantly larger for only-vdW cases than that for Casimir-vdW cases. Interestingly enough, $q_{\text {PI }}$ for simulation set (ii) (see figure $3 b$ ) is almost an order of magnitude greater than that for simulation set (i) (see figure $3 a$ ). This may be explained on the basis of higher bending stiffness and shorter length for the nano beam used in simulation set (ii). Assuming that both van der Waals and Casimir forces can be modelled as uniformly distributed loads (UDL), basic principles of deformable beams give that the deflection at the free end of a cantilever beam subjected to a UDL is $\sim L^{4} / Y I$. Here $Y$ equals Young's modulus of the beam material, and $I$ its second moment of area about the centroidal axis. Thus, the ratio of deflection of the nano-beam used in simulation set (ii) to that of the nano beam used in simulation set (i) is 0.0324 . Higher deflection under a given load suggests that the nano-beam of simulation set (i) gets pulled in at a smaller charge.

Typically, the displacement, $y$, of a point of a continuum cantilever nano-beam is governed by the Euler-Bernoulli beam theory equation [78]

$$
Y I \frac{d^{4} y}{d x^{4}}=f_{\text {elec }}+f_{\text {disp }}
$$

where $Y I$ represents the bending stiffness of the nano-beam, and $f_{\text {elec }}$ and $f_{\text {disp }}$ represent, respectively, the electrostatic (Coulomb) and the van der Waals/Casimir forces per unit length. Mathematically, these forces may be written as [78]

$$
f_{\text {elec }}=\frac{\epsilon_{0} w V^{2}}{2(g-y)^{2}}\left(1+0.65\left(\frac{g-y}{w}\right)\right)
$$

and

$$
f_{\text {disp }}= \begin{cases}\frac{A_{H} w}{6 \pi(g-y)^{3}} & \text { for van der Waals interaction. } \\ \frac{\pi^{2} h c w}{240(g-y)^{4}} & \text { for Casimir interaction. }\end{cases}
$$

Here, $V$ is the voltage applied, $w$ the width of the beam and $\epsilon_{0}$ the vacuum permittivity, with the remaining variables having the same meaning as described before. The interested readers are referred to [78] for the solution of equation (4.1).

Defining $\beta=\epsilon_{0} w V^{2} L^{4} / 2 g^{3} Y I$, the pull-in voltage, $V_{\mathrm{PI}}$, can be obtained by solving the equation $\partial \beta / \partial y=0$. In order to make a comparison between the MD and analytical models, 
Table 1. Comparison of results obtained from our MD simulations $\left(Q_{m d}\right)$ with those obtained from continuum scale theory $\left(Q_{t h}\right)$. Note that for calculating $Q_{\text {th }}$, elastic modulus, $Y$, has been assumed to be $175 \mathrm{GPa}$. While there is a qualitative agreement between the results $-Q$ decreases for simulation set (ii) in comparison to that for set (i), quantitatively the results differ significantly. The large deviation arises because - (a) the Casimir energy formulation is for infinite plates, whereas in MD, the dimensions of the graphene sheet are finite, $(b) Q_{\text {th }}$ either accounts for the Casimir $\left(\beta_{\text {cas }}\right)$ or the van der Waals forces $\left(\beta_{\mathrm{vdw}}\right)$, but not for the transition from the Casimir to the van der Walls, while $Q_{M D}$ accounts for this transition, and (c) the computation of the macroscopic Casimir potential for non-metals like silicon involves a correction term that has been omitted for simplicity.

\begin{tabular}{llll} 
set & $Q_{\text {th }}$ & $Q_{\text {md }}$ & deviation \\
simulation set (i) & 1.055 & 2.0 & $89 \%$ \\
\hline simulation set (ii) & 1.041 & 1.6 & $54 \%$ \\
\hline
\end{tabular}

$\beta$ corresponding to pull-in is found separately when only the van der Waals forces act $\left(\beta_{\mathrm{vdW}}\right)$ and when only the Casimir forces act $\left(\beta_{\mathrm{cas}}\right)$. Their ratio: $Q_{\mathrm{th}}=\sqrt{\beta_{\mathrm{vdW}} / \beta_{\mathrm{cas}}}$ is compared with $Q_{\mathrm{md}}$, and the results are shown in table 1 . While there is a qualitative agreement between the results$Q$ decreases for simulation set (ii) in comparison to that for set (i), quantitatively the results differ significantly. The difference may be attributed to the fact that-(a) our MD model incorporates a switch to shift between van der Waals and Casimir forces depending on the distance between the electrode and the substrate, while analytical models employ either van der Waals forces or Casimir forces for all values of separation between the electrode and the substrate, (b) continuum scale model of van der Waals and electrostatic forces have been developed under the assumption that both electrode and substrate are infinite in one of the dimensions, and (c) assumptions of the Euler-Bernoulli beam theory do not hold for modelling a very short nano-beam as has been used in the present study.

\section{(b) Transient and steady-state dynamics of electrode comprised of cantilever carbon nanotube}

We now study the dynamics—-both transient and steady state—of a cantilever NEMS made of a CNT. Due to the difference in geometry with respect to the rectangular beam along with a different number density of atoms, quantitatively the dynamics of CNT NEMS is different from that of the previous case investigated. However, qualitatively the dynamics remains similar.

The essential features of the dynamics at a temperature of $1 \mathrm{~K}$ in presence/absence of the Casimir force modelled under the PFA (see equation (2.11)) are shown in figure 4: (a) plots the temporal history of the free-end tip for two specific values of charge, and (b) the steady-state and minimum location of the free-end tip as the charge increases. It is evident from figure $4 a$ that the maximum tip displacement occurs at the initial stages. Following the initial transients, the tip vibration reaches a steady state. The steady-state location of the tip is taken as the average displacement over the last 100000 time steps. The problem is analogous to a cantilever beam undergoing a step loading in a damped environment. In our case, the sudden increment in charge, which remains constant thereafter, acts like a step loading, whereas the simulation being carried out at a constant temperature of $1 \mathrm{~K}$ acts like the damped environment. Note that the energy from the CNT is extracted by the heat reservoir here. In view of these transients, there is a disparity between the minimum and the steady-state location of a tip post charge increment, which increases with increasing charge. These results can be seen clearly in figure $4 b$. Unsurprisingly, both the minimum and the steady-state tip locations are closer to the substrate when one considers the Casimir-vdW case. Because of its attractive nature, the Casimir force supplements the attractive electrostatic force causing larger displacements than in the only-vdW case, where only the van der Waals forces are present. Note that the difference in the minimum tip location between the two cases (Casimir-vdW and only-vdW) is around $0.10-0.14 \mathrm{~nm}$, while that of the steady-state tip location is between $0.10-0.13 \mathrm{~nm}$. It is likely that the differences would be 

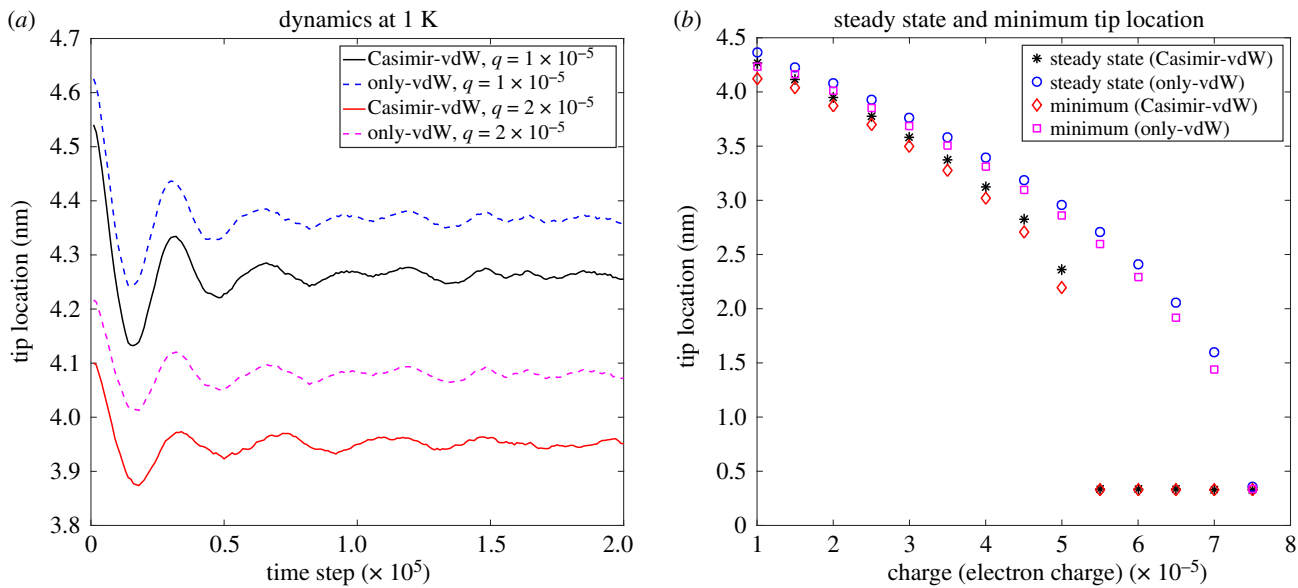

Figure 4. The dynamics at $1 \mathrm{~K}$ of a CNT cantilever nano-beam: (a) the transient and steady-state dynamics of the tip of the cantilever beam in presence/absence of the Casimir force for two different values of charge per atom, and $(b)$ the minimum and the steady-state location of the free-end tip of the cantilever beam. For Casimir-vdW cases - (i) the cantilever tip has larger steady state and initial transient displacements, and (ii) relatively smaller charge per atom causes pull-in instability vis-á-vis when only-vdW cases.

greater in larger MEMS and NEMS devices having dimensions two orders of magnitude greater than those in the present case.

The dynamics is substantially different at $300 \mathrm{~K}$ when compared with those at $1 \mathrm{~K}$, as can be seen in figure 5: (a) shows the instantaneous location of the tip for the cases-Casimir-vdW and only-vdW-with two specific values of charge per atom, and (b) plots the minimum and the steady-state tip location for the two cases as the charge per atom increases. Note that in this case as well, the Casimir forces are modelled through the short-ranged PFA potential (see equation (2.11)), so that $C=C_{P F A}$. Interestingly, unlike that for the $1 \mathrm{~K}$, the minimum tip location does not occur at the initial stages at $300 \mathrm{~K}$. This may be attributed to the relatively large random thermal forces at $300 \mathrm{~K}$ that offset the initial step loading occurring due to charge increment. At $300 \mathrm{~K}$, the pull-in instability occurs at a relatively smaller value of the charge per atom than that at $1 \mathrm{~K}$-in presence of the Casimir and the van der Waals forces, the pull-in occurs at $5.0 \times 10^{-5} \mathrm{e}$ at $300 \mathrm{~K}$ and at $5.5 \times 10^{-5}$ e at $1 \mathrm{~K}$. Irrespective of the thermal environment, the results suggest that we consider Casimir forces and its switch to van der wall forces till pull-in occurs.

\section{(c) Effect of longer-ranged Casimir interactions}

As has been discussed before, our proposed framework of incorporating Casimir interactions in MD depends on matching the energy obtained through pair-wise summation with that obtained from continuum scale calculations. Changing the continuum energy alters the constant $C$ because of which MD results change as well. For plate-cylinder geometry, one can obtain two different constants- $-C_{\mathrm{PFA}}$ and $C_{\mathrm{LOG}}$ (see equations (2.11) and (2.12)) - depending on whether the PFA is made or not. Since the Casimir force is known to be among the forces that have the slowest possible decay with distance, for designing NEMS, where the effect of Casimir forces is significant, it makes sense to have a parametric study on $C$.

The electrostatic potential decreases as $r^{-1}$, and while the two Casimir potentials (LOG and PFA) have a complex dependence on the distance (since $C$ is no longer a constant), they necessarily fall faster than $r^{-1}$. Thus, it is not surprising that for every separation distance, the Casimir potential is only a fraction of the electrostatic potential. This is depicted in figure 6 for four different charges at $1 \mathrm{~K}$. With increasing charge, the electrostatic potential increases as well. However, the increase in the Casimir potential is not commensurate, and with increasing 

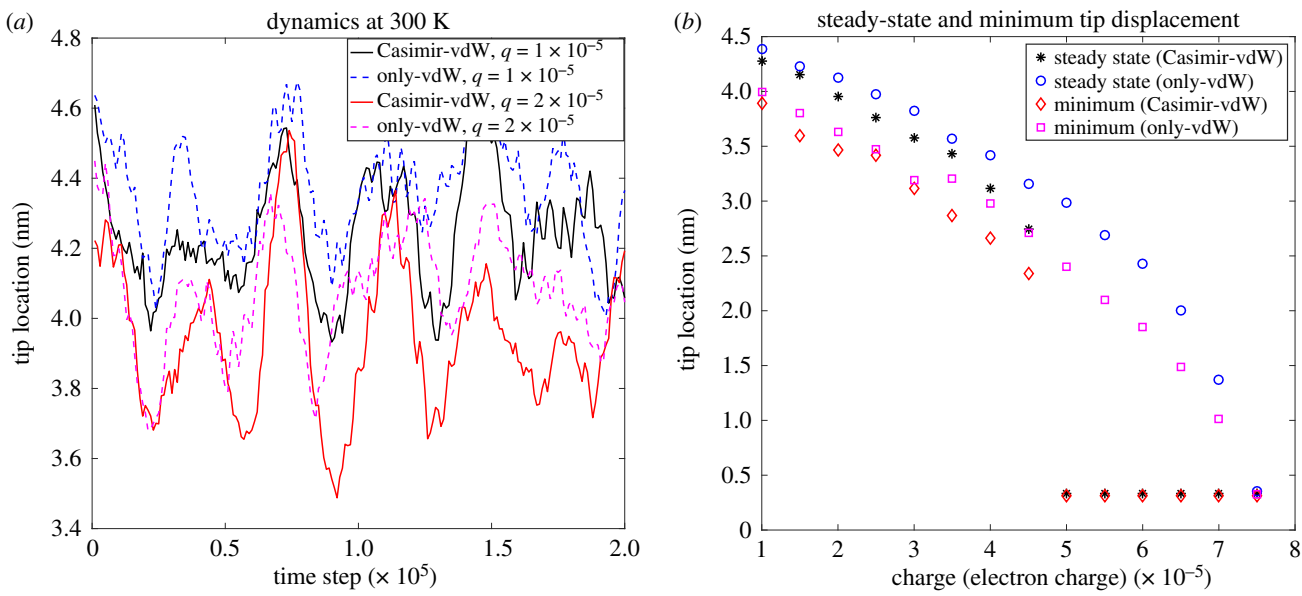

Figure 5. The dynamics at $300 \mathrm{~K}$ of a CNT cantilever nano-beam : (a) the transient and the steady-state dynamics of the tip of the cantilever beam in the presence/absence of Casimir forces for two different values of charge per atom, and $(b)$ the minimum and the steady-state location of the free-end tip of the cantilever beam. The temporal evolution of the tip at $300 \mathrm{~K}$ is substantially different from that at $1 \mathrm{~K}$, primarily because of the random thermal forces. However, like in the $1 \mathrm{~K}$ case, for Casimir-vdW cases, a relatively smaller charge per atom causes pull-in instability vis-á-vis only-vdW cases. (Online version in colour.)

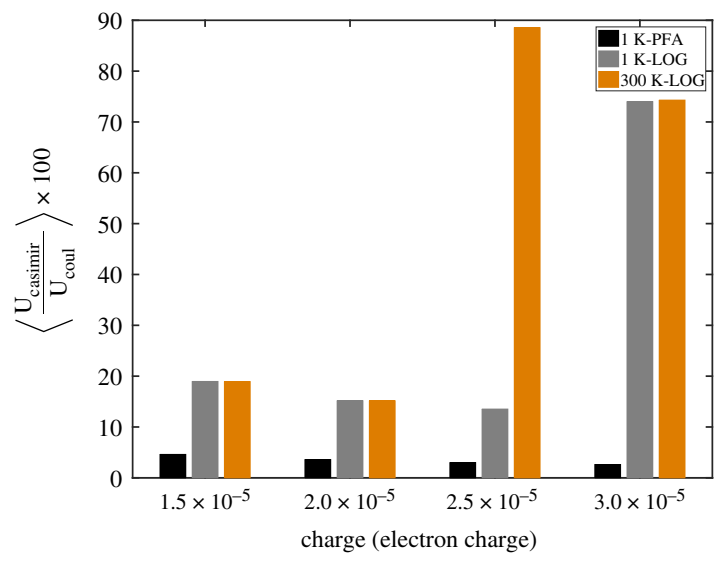

Figure 6. Relative contribution of the Casimir and the electrostatic potentials. The LOG potential contributes more than the PFA potential because of its longer-ranged nature. With increasing charge, until the pull-in instability, the relative contribution of the Casimir potential decreases. This is because the electrostatic potential varies as $1 / r$, while the Casimir potential falls off relatively faster than $1 / r$. Once the pull-in instability occurs, there is a sudden rise in the relative contribution of the Casimir potential. (Online version in colour.)

charge (until the pull-in), the contribution of the Casimir potential decreases. Interestingly, the contribution of the LOG potential is more than double that of the PFA potential until the pull-in occurs, which is marked by a sudden increase in the contribution of the Casimir potential vis-á-vis the electrostatic potential.

Figure 7 depicts the effect of using the longer-ranged LOG potential (equation (2.12)) as against the shorter-ranged PFA potential (equation (2.11)) on the charge necessary to cause the pull-in instability at two different temperatures. The pull-in charge significantly depends on the range of the potential - compared to the shorter-ranged PFA potential, the pull-in charge is smaller for the longer-ranged LOG potential. This reduction is observed at both temperatures. At $1 \mathrm{~K}$, 


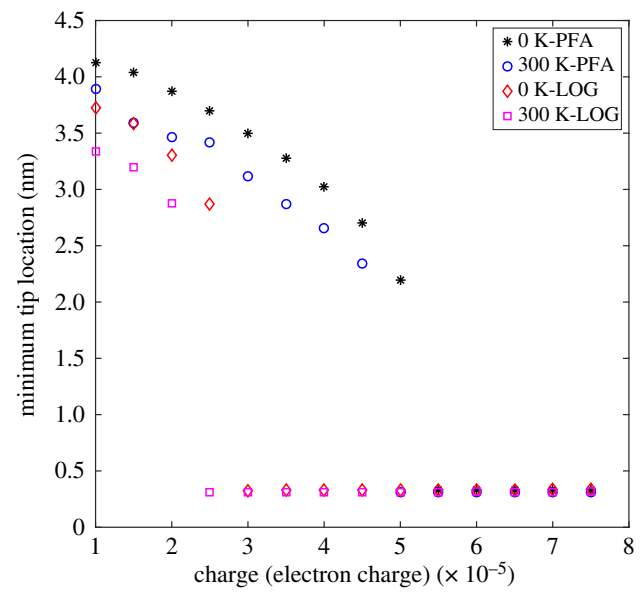

Figure7. Effect of using the longer-ranged LOG potential as against the shorter-ranged PFA potential on the charge necessary to cause the pull-in instability at $1 \mathrm{~K}$ and $300 \mathrm{~K}$. The pull-in charge significantly depends on the range of the potential — compared to the shorter-ranged PFA potential, the pull-in charge is smaller for the longer-ranged LOG potential. This reduction is observed at both temperatures studied here. (Online version in colour.)
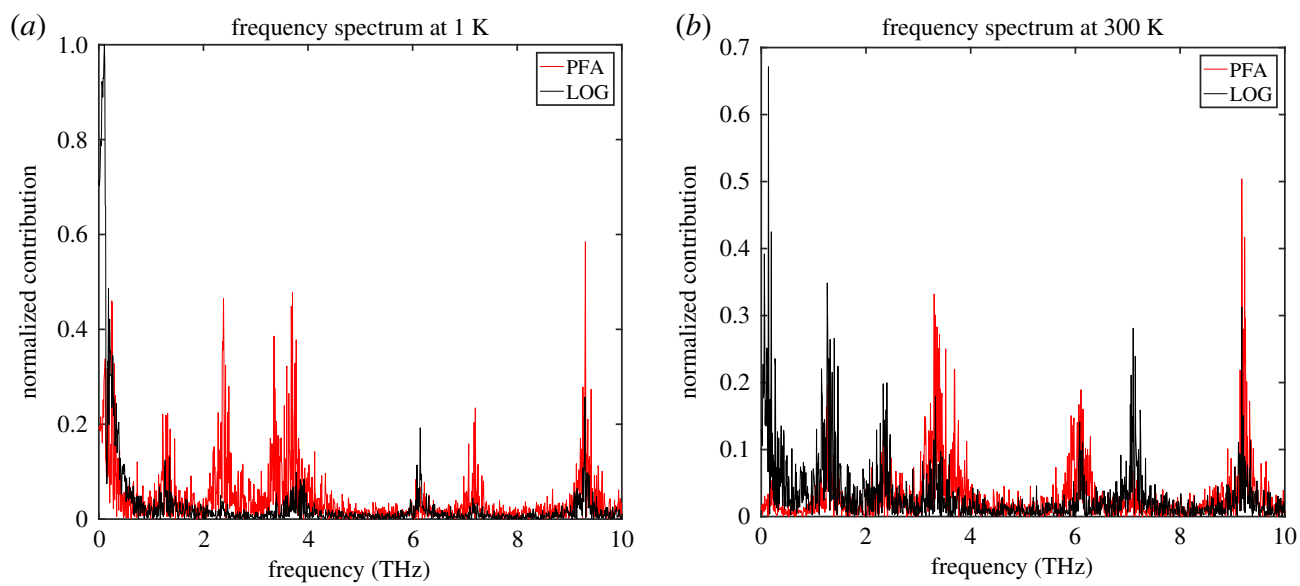

Figure 8. Frequency spectrum of the CNT cantilever nano-beam at two different temperatures: (a) at $1 \mathrm{~K}$ and $(b)$ at $300 \mathrm{~K}$. The Casimir effect has been modelled separately through the PFA and the LOG potentials. Note the similarity between the frequency spectra for the two potentials at both temperatures. (Online version in colour.)

while for the PFA potential the electrode collapses at a charge of $5.5 \times 10^{-5} \mathrm{e}$, the same occurs at $3 \times 10^{-5} \mathrm{e}$ for the LOG potential-a reduction of almost $50 \%$. Likewise, a reduction of approximately $50 \%$ is observed at $300 \mathrm{~K}$ temperature as well. An important consequence of these results is that the longer-ranged potential must be adopted while designing NEMS. However, incorporation of the longer-ranged forces results in almost $20 \%$ increase in the computational time for the analysis. This is primarily because of two reasons-longer-range forces imply a larger cutoff distance that substantially increases the number of neighbours in the neighbour list, and the evaluation of the natural logarithm at every time step.

When it comes to the frequency response spectra, the range of the Casimir forces do not play a significant role as can be seen from figure 8 . The figure plots the normalized contribution of different modes of vibration for the PFA and the LOG type Casimir interactions at the two temperatures. The vibrational frequency has been obtained by taking the Fourier transform of 


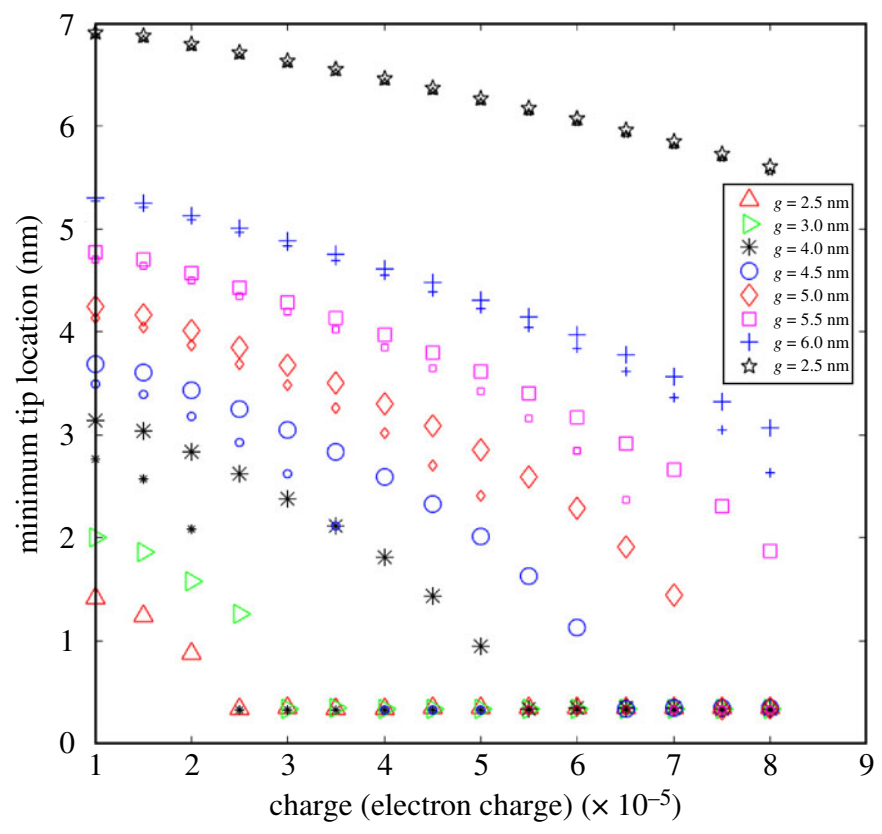

Figure 9. The minimum location of the free-end tip with increasing separation gap, $g$. The larger markers correspond to results without the Casimir interaction while the smaller ones are for the PFA type Casimir interaction. Note the increasing disparity between the two sets of results as the charge per atom increases. (Online version in colour.)

the velocity auto-correlation function. In both methods, the different frequencies hit peak at almost the same value for both temperatures. Thus, for NEMS devices used in signal filtering, and high sensitivity chemical and mass sensing that typically rely on change in frequencies, it is not necessary to adopt the longer-ranged LOG potential. The main advantage of using the PFA potential here is savings in the computational time.

\section{(d) Pull-in charge and displacement}

We now study the role of the PFA type Casimir potential on the pull-in charge and displacement as the initial separation between the electrode and the substrate increases. Notice that LOG type Casimir potential has not been investigated owing to the larger computational time requirement. Figure 9 shows the minimum tip location of the free end of the cantilever CNT as the charge per atom on the substrate increases. The larger markers correspond to results of only-vdW cases while the smaller ones are for the Casimir-vdW cases with PFA type Casimir interactions. The figure reveals that even in the absence of any electrical charges on atoms of the substrate and the electrode, the tip deflects due to the presence of dispersion forces. The most pronounced effect is seen in the presence of the Casimir forces at small separation distances- $g=2.5 \mathrm{~nm}$ and $3.0 \mathrm{~nm}$ - where the nanotubes undergo pull-in even in the absence of any charge. Since pull-in occurs without applying $q$, it provides the limiting value of $g$ for fabricability of this NEMS as was pointed out by Batra et al. [79] who used a continuum theory. With increasing charge, the disparity between results of the presence/absence of the Casimir forces increases. This is because the nanotube deflection increases progressively under the electrostatic forces, which in turn increases the Casimir forces. The increased Casimir forces, being attractive, brings the tip closer to the substrate. As expected, with increasing separation distances the pull-in instability occurs at a larger value of applied charges. The increasing difference of the tip displacement as the voltage increases and the reduction in pull-in charge with decreasing separation are in 
qualitative agreement with the previously reported results (see $[43,78])$. We conjecture that the pull-in potential would further decrease if one uses the LOG potential.

\section{Conclusion and the path forward}

Casimir forces are expected to play a very important role in nanoscale devices as they are longer ranged than the traditional van der Waals forces. Being longer ranged, these forces can cause unexpected performance in devices if not considered in the design/fabrication phases. While Casimir forces have been accounted for in several analytical studies based on continuum modelling, atomistic scale treatment of Casimir forces is lacking in the literature. In this work, we remedy the situation by proposing a methodology for including Casimir forces within the framework of MD simulations. The developed methodology is limited to nanoscale devices that have plate-rectangular and plate-cylinder geometries, but with some modifications, can be successfully employed for other geometries as well.

The proposed methodology relies on constructing an appropriate form of atomistic pair-wise Casimir potential, which is then considered within the traditional MD framework. For modelling Casimir interactions, we assume that an atom pair interacts through a potential of the form $C / r^{7}$. After summing up the contributions arising from all atom pairs, the value of the constant $C$ is obtained by matching the summed-up pair-wise energy with the total energy obtained using continuum scale calculations. In other words, $C$ is chosen such that the Casimir energy obtained by summing up the pair-wise interactions is the same as that obtained by using a continuum theory. Using this assumption, we have found different values of $C$ for plate-rectangular and plate-cylinder geometries.

The proposed methodology has been used to study effects of Casimir forces on pull-in instability of cantilever NEMS devices. Our results for plate-rectangular geometry qualitatively agree with those from continuum scale analyses. Quantitatively, however, there is a significant mismatch, primarily because: (a) our MD model allows for switching from the Casimir potential to the van der Waals potential when the separation between atoms of the electrode and the substrate crosses a predefined limiting value, while analytical models employ either van der Waals forces or Casimir forces, (b) continuum scale models of van der Waals and electrostatic forces have been developed under the assumption that both the electrode and the substrate are infinite in one of the dimensions, and (c) the assumptions of the continuum theory may not be valid for modelling a very short nano-beam. With the continuous miniaturization of devices, the role played by the Casimir forces is increasing. We now have devices in nano-metre scale as well. At these scales, the use of continuum analytical expressions for understanding the effects of the Casimir forces is fraught with difficulties, and a fully atomistic treatment is necessary. While analytical techniques account for either the van der Waals forces or the Casimir forces but not both, the primary benefits of the proposed methodology are: (a) the distance-based switch that automatically shifts between the Casimir and the van-der-Waals forces and (b) a fully atomistic treatment of the problem.

Our results indicate the significant role played by the Casimir forces-the free-end tip of the cantilever beam undergoes larger displacement in the presence of Casimir forces than in its absence, causing the beam to undergo pull-in at smaller charges. Furthermore, the range of Casimir forces plays a significant role in determining the pull-in charges-incorporating the longer-ranged LOG potential instead of the shorter-ranged PFA potential increases the free-end tip displacement at the same potential difference. The charge required for pull-in is smaller at $300 \mathrm{~K}$ than that at $1 \mathrm{~K}$. With the LOG potential, the reduction in the charge could be as large as $50 \%$ over that for the PFA potential, and $67 \%$ above that for the van der Waals interactions. However, incorporating the LOG potential comes at a price-being longer ranged, it is computationally more expensive than the PFA potential. When it comes to understanding the vibrational spectra at the two temperatures, changing from the LOG to the PFA potential does not significantly alter the frequency response spectrum. 
There is a scope of further improving the proposed methodology. In reality, Casimir forces are non-additive and the presence of a third particle alters the interaction between two particles [63]. Consequently, two-body interaction terms, in general, are insufficient and this could be a reason for the disparity between our results and the analytically computed Casimir potential. A way out could be developing multibody potentials using the Lifshitz theory. In addition, Casimir forces have a strong geometry dependence. Thus, as a nano-beam deforms, the expression of the continuum Casimir potential changes. This is significantly difficult to tackle as expressions have been developed only for simple geometries. The present work sets up a good stage to further explore Casimir effects occurring in atomic scale systems, and may be used by engineers to design NEMS devices.

Data accessibility. The codes for the different figures are available on GitHub: https://github.com/puneetpatra/ Casimir_LAMMPS.git.

Authors' contributions. P.K.P. and R.C.B. conceived the problem which was implemented by P.K.P. and A.S. All three authors contributed to writing the work. All authors gave final approval for publication and agree to be held equally accountable for the work performed therein.

Competing interests. The authors do not have any competing interests.

Funding. Support for the research provided in part by Indian Institute of Technology Kharagpur under the grant DNI is gratefully acknowledged by P.K.P. P.K.P. also acknowledges the computational support provided as a part of the DST-SERB grant no. ECR/2018/000340.

Acknowledgements. A.S. and P.K.P. thank Centre for Development of Advanced Computing (CDAC), Pune for permission to use PARAM Yuva II, where a part of the simulations was carried out.

\section{References}

1. Casimir HB. 1948 On the attraction between two perfectly conducting plates. Front. Phys. 100, 61-63.

2. Derjaguin B, Abrikosova I, Lifshitz E. 1956 Direct measurement of molecular attraction between solids separated by a narrow gap. Q. Rev., Chem. Soc. 10, 295-329. (doi:10.1039/ qr9561000295)

3. Boström M, Sernelius BE, Brevik I, Ninham BW. 2012 Retardation turns the van der Waals attraction into a Casimir repulsion as close as $3 \mathrm{~nm}$. Phys. Rev. A 85, 010701. (doi:10.1103/PhysRevA.85.010701)

4. Lamoreaux SK. 1999 Resource letter CF-1: Casimir force. Am. J. Phys. 67, 850-861. (doi:10.1119/1.19138)

5. Bordag M, Mohideen U, Mostepanenko VM. 2001 New developments in the Casimir effect. Phys. Rep. 353, 1-205. (doi:10.1016/S0370-1573(01)00015-1)

6. Klimchitskaya G, Mohideen U, Mostepanenko V. 2000 Casimir and van der Waals forces between two plates or a sphere (lens) above a plate made of real metals. Phys. Rev. A 61, 062107. (doi:10.1103/PhysRevA.61.062107)

7. Batra R, Porfiri M, Spinello D. 2008 Reduced-order models for microelectromechanical rectangular and circular plates incorporating the Casimir force. Int. J. Solids Struct. 45, 3558-3583. (doi:10.1016/j.ijsolstr.2008.02.019)

8. Israelachvili J, Tabor D. 1972 Measurement of van der Waals dispersion forces in the range 1.4 to $130 \mathrm{~nm}$. Nat. Phys. Sci. 236, 106-106. (doi:10.1038/physci236106a0)

9. Hertzberg M, Jaffe R, Kardar M, Scardicchio A. 2005 Attractive Casimir forces in a closed geometry. Phys. Rev. Lett. 95, 250402. (doi:10.1103/PhysRevLett.95.250402)

10. Neto PAM, Lambrecht A, Reynaud S. 2008 Casimir energy between a plane and a sphere in electromagnetic vacuum. Phys. Rev. A 78, 012115. (doi:10.1103/PhysRevA.78.012115)

11. Casimir HB, Polder D. 1948 The influence of retardation on the London-van der Waals forces. Phys. Rev. 73, 360. (doi:10.1103/PhysRev.73.360)

12. Emig T. 2008 Fluctuation-induced quantum interactions between compact objects and a plane mirror. J. Stat. Mech: Theory Exp. 2008, P04007. (doi:10.1088/1742-5468/2008/04/P04007)

13. Rahi SJ, Emig T, Jaffe RL, Kardar M. 2008 Casimir forces between cylinders and plates. Phys. Rev. A 78, 012104. (doi:10.1103/PhysRevA.78.012104)

14. Emig T, Jaffe R, Kardar M, Scardicchio A. 2006 Casimir interaction between a plate and a cylinder. Phys. Rev. Lett. 96, 080403. (doi:10.1103/PhysRevLett.96.080403) 
15. Lambrecht A, Neto PAM, Reynaud S. 2006 The Casimir effect within scattering theory. New J. Phys. 8, 243. (doi:10.1088/1367-2630/8/10/243)

16. Gupta RK, Senturia SD. 1997 Pull-in time dynamics as a measure of absolute pressure. In Proc. IEEE The Tenth Annual Int. Workshop on Micro Electro Mechanical Systems. An Investigation of Micro Structures, Sensors, Actuators, Machines and Robots, pp. 290-294. Piscataway, NJ: IEEE.

17. Hung ES, Senturia SD. 1999 Extending the travel range of analog-tuned electrostatic actuators. J. Microelectromech. Syst. 8, 497-505. (doi:10.1109/84.809065)

18. Nguyen CC, Katehi LP, Rebeiz GM. 1998 Micromachined devices for wireless communications. Proc. IEEE 86, 1756-1768. (doi:10.1109/5.704281)

19. Kwon YK, Tománek D, Iijima S. 1999 `Bucky Shuttle’ memory device: synthetic approach and molecular dynamics simulations. Phys. Rev. Lett. 82, 1470. (doi:10.1103/PhysRevLett.82.1470)

20. Fruehling A, Xiao S, Qi M, Roy K, Peroulis D. 2009 Nano-switch for study of gold contact behavior. In SENSORS, 2009 IEEE, pp. 248-251. Piscataway, NJ: IEEE.

21. Pelesko JA, Bernstein DH. 2002 Modeling mems and nems. Boca Raton, FL: CRC Press.

22. Pelesko J, Bernstein D, McCuan J. 2003 Symmetry and symmetry breaking in electrostatic MEMS. preprint.

23. Younis MI, Abdel-Rahman EM, Nayfeh A. 2003 A reduced-order model for electrically actuated microbeam-based MEMS. J. Microelectromech. Syst. 12, 672-680. (doi:10.1109/JMEMS. 2003.818069)

24. Nayfeh AH, Younis MI. 2005 Dynamics of MEMS resonators under superharmonic and subharmonic excitations. J. Micromech. Microeng. 15, 1840. (doi:10.1088/0960-1317/ $15 / 10 / 008)$

25. Chiu HY, Hung P, Postma HWC, Bockrath M. 2008 Atomic-scale mass sensing using carbon nanotube resonators. Nano Lett. 8, 4342-4346. (doi:10.1021/nl802181c)

26. Taylor GI. 1968 The coalescence of closely spaced drops when they are at different electric potentials. Proc. R. Soc. A 306, 423-434. (doi:10.1098/rspa.1968.0159)

27. Nathanson HC, Newell WE, Wickstrom RA, Davis JR. 1967 The resonant gate transistor. IEEE Trans. Electron Devices 14, 117-133. (doi:10.1109/T-ED.1967.15912)

28. Legtenberg R, Tilmans HA. 1994 Electrostatically driven vacuum-encapsulated polysilicon resonators Part I. Design and fabrication. Sens. Actuators, A 45, 57-66. (doi:10.1016/ 0924-4247(94)00812-4)

29. Tilmans HA, Legtenberg R. 1994 Electrostatically driven vacuum-encapsulated polysilicon resonators: Part II. Theory and performance. Sens. Actuators, A 45, 67-84. (doi:10.1016/0924-4247(94)00813-2)

30. Pelesko J, Triolo A. 2001 Nonlocal problems in MEMS device control. J. Eng. Math. 41, 345-366. (doi:10.1023/A:1012292311304)

31. Francais O, Dufour I. 1999 Normalized abacus for the global behavior of diaphragms: pneumatic, electrostatic, piezoelectric or electromagnetic actuation. J. Model. Simul. Microsyst. $2,149-160$.

32. Ng T, Jiang T, Li H, Lam K, Reddy J. 2004 A coupled field study on the nonlinear dynamic characteristics of an electrostatic micropump. J. Sound Vib. 273, 989-1006. (doi:10.1016/S0022-460X(03)00517-0)

33. Zhao X, Abdel-Rahman EM, Nayfeh AH. 2004 A reduced-order model for electrically actuated microplates. J. Micromech. Microeng. 14, 900. (doi:10.1088/0960-1317/14/7/009)

34. Batra R, Porfiri M, Spinello D. 2007 Review of modeling electrostatically actuated microelectromechanical systems. Smart Mater. Struct. 16, R23. (doi:10.1088/0964-1726/ 16/6/R01)

35. Porfiri M. 2008 Vibrations of parallel arrays of electrostatically actuated microplates. J. Sound Vib. 315, 1071-1085. (doi:10.1016/j.jsv.2008.02.007)

36. Wang K, Kitamura T, Wang B. 2015 Nonlinear pull-in instability and free vibration of micro/nanoscale plates with surface energy-a modified couple stress theory model. Int. J. Mech. Sci. 99, 288-296. (doi:10.1016/j.ijmecsci.2015.05.006)

37. Wang K, Wang B, Zhang C. 2017 Surface energy and thermal stress effect on nonlinear vibration of electrostatically actuated circular micro-/nanoplates based on modified couple stress theory. Acta Mech. 228, 129-140. (doi:10.1007/s00707-016-1701-7)

38. Pelesko JA. 2002 Mathematical modeling of electrostatic MEMS with tailored dielectric properties. SIAM J. Appl. Math. 62, 888-908. (doi:10.1137/S0036139900381079)

39. Pelesko J, Chen X. 2003 Electrostatic deflections of circular elastic membranes. J. Electrostat. 57, 1-12. (doi:10.1016/S0304-3886(02)00073-6) 
40. Batra RC, Porfiri M, Spinello D. 2006 Analysis of electrostatic MEMS using meshless local Petrov-Galerkin (MLPG) method. Eng. Anal. Boundary Elem. 30, 949-962. (doi:10.1016/ j.enganabound.2006.04.008)

41. Yang J, Jia X, Kitipornchai S. 2008 Pull-in instability of nano-switches using nonlocal elasticity theory. J. Phys. D: Appl. Phys. 41, 035103. (doi:10.1088/0022-3727/41/3/035103)

42. Ramezani A, Alasty A, Akbari J. 2006 Influence of van der Waals force on the pullin parameters of cantilever type nanoscale electrostatic actuators. Microsyst. Technol. 12, 1153-1161. (doi:10.1007/s00542-006-0244-6)

43. Ramezani A, Alasty A, Akbari J. 2007 Closed-form solutions of the pull-in instability in nano-cantilevers under electrostatic and intermolecular surface forces. Int. J. Solids Struct. 44, 4925-4941. (doi:10.1016/j.ijsolstr.2006.12.015)

44. Farrokhabadi A, Abadian N, Rach R, Abadyan M. 2014 Theoretical modeling of the Casimir force-induced instability in freestanding nanowires with circular cross-section. Physica E 63, 67-80. (doi:10.1016/j.physe.2014.05.015)

45. Wang K, Wang B. 2015 A general model for nano-cantilever switches with consideration of surface effects and nonlinear curvature. Physica E 66, 197-208. (doi:10.1016/j.physe. 2014.10.012)

46. Mokhtari J, Farrokhabadi A, Rach R, Abadyan M. 2015 Theoretical modeling of the effect of Casimir attraction on the electrostatic instability of nanowire-fabricated actuators. Physica $E$ 68, 149-158. (doi:10.1016/j.physe.2014.12.003)

47. Farrokhabadi A, Mokhtari J, Rach R, Abadyan M. 2015 Modeling the influence of the Casimir force on the pull-in instability of nanowire-fabricated nanotweezers. Int. J. Mod. Phys. B 29, 1450245. (doi:10.1142/S0217979214502452)

48. Farrokhabadi A, Rach R, Abadyan M. 2013 Modeling the static response and pull-in instability of CNT nanotweezers under the Coulomb and van der Waals attractions. Physica E 53, 137-145. (doi:10.1016/j.physe.2013.05.003)

49. Wang K, Zeng S, Wang B. 2017 Large amplitude free vibration of electrically actuated nanobeams with surface energy and thermal effects. Int. J. Mech. Sci. 131, 227-233. (doi:10.1016/j.ijmecsci.2017.06.049)

50. Pamidighantam S, Puers R, Baert K, Tilmans HA. 2002 Pull-in voltage analysis of electrostatically actuated beam structures with fixed-fixed and fixed-free end conditions. J. Micromech. Microeng. 12, 458. (doi:10.1088/0960-1317/12/4/319)

51. Chu PB, Nelson PR, Tachiki ML, Pister KS. 1996 Dynamics of polysilicon parallelplate electrostatic actuators. Sens. Actuators, A 52, 216-220. (doi:10.1016/0924-4247(96) 80152-X)

52. Batra RC, Porfiri M, Spinello D. 2006 Electromechanical model of electrically actuated narrow microbeams. J. Microelectromech. Syst. 15, 1175-1189. (doi:10.1109/JMEMS.2006.880204)

53. Batra R, Porfiri M, Spinello D. 2008 Vibrations of narrow microbeams predeformed by an electric field. J. Sound Vib. 309, 600-612. (doi:10.1016/j.jsv.2007.07.030)

54. Serry FM, Walliser D, Maclay GJ. 1998 The role of the Casimir effect in the static deflection and stiction of membrane strips in microelectromechanical systems (MEMS). J. Appl. Phys. 84, 2501-2506. (doi:10.1063/1.368410)

55. Lin WH, Zhao YP. 2005 Casimir effect on the pull-in parameters of nanometer switches. Microsyst. Technol. 11, 80-85. (doi:10.1007/s00542-004-0411-6)

56. Batra R, Porfiri M, Spinello D. 2008a Vibrations and pull-in instabilities of microelectromechanical von Kármán elliptic plates incorporating the Casimir force. J. Sound Vib. 315, 939-960. (doi:10.1016/j.jsv.2008.02.008)

57. Batra R, Porfiri M, Spinello D. 2008b Reduced-order models for microelectromechanical rectangular and circular plates incorporating the Casimir force. Int. J. Solids Struct. 45, 3558-3583. (doi:10.1016/j.ijsolstr.2008.02.019)

58. Lu P, Lee H, Lu C, Zhang P. 2007 Application of nonlocal beam models for carbon nanotubes. Int. J. Solids Struct. 44, 5289-5300. (doi:10.1016/j.ijsolstr.2006.12.034)

59. Dequesnes M, Rotkin S, Aluru NR. 2002 Calculation of pull-in voltages for carbonnanotube-based nanoelectromechanical switches. Nanotechnology 13, 120. (doi:10.1088/0957$4484 / 13 / 1 / 325)$

60. Fakhrabadi MMS, Khorasani PK, Rastgoo A, Ahmadian MT. 2013 Molecular dynamics simulation of pull-in phenomena in carbon nanotubes with Stone-Wales defects. Solid State Commun. 157, 38-44. (doi:10.1016/j.ssc.2012.12.016) 
61. Hwang Z, Lee JH, Kang JW. 2013 Molecular dynamics study on graphene-based nanoelectromechanical relays. J. Comput. Theor. Nanosci. 10, 1892-1898. (doi:10.1166/ jctn.2013.3145)

62. Kenneth O, Klich I, Mann A, Revzen M. 2002 Repulsive Casimir forces. Phys. Rev. Lett. 89, 033001. (doi:10.1103/PhysRevLett.89.033001)

63. Milonni PW, Shih ML. 1992 Casimir forces. Contemp. Phys. 33, 313-322. (doi:10.1080/ 00107519208223981)

64. Fosco CD, Lombardo FC, Mazzitelli FD. 2011 Proximity force approximation for the Casimir energy as a derivative expansion. Phys. Rev. D 84, 105031. (doi:10.1103/PhysRevD.84.105031)

65. Brothers EN, Izmaylov AF, Scuseria GE, Kudin KN. 2008 Analytically calculated polarizability of carbon nanotubes: single wall, coaxial, and bundled systems. J. Phys. Chem. C 112, 1396-1400. (doi:10.1021/jp709931r)

66. Srivastav S, Xu C, Edström K, Gustafsson T, Brandell D. 2017 Modelling the morphological background to capacity fade in Si-based lithium-ion batteries. Electrochim. Acta 258, 755-763. (doi:10.1016/j.electacta.2017.11.124)

67. Gusso A, Delben G. 2007 Influence of the Casimir force on the pull-in parameters of silicon based electrostatic torsional actuators. Sens. Actuators, A 135, 792-800. (doi:10.1016/ j.sna.2006.09.008)

68. Tersoff J. 1989 Modeling solid-state chemistry: interatomic potentials for multicomponent systems. Phys. Rev. B 39, 5566. (doi:10.1103/PhysRevB.39.5566)

69. Lindsay L, Broido D. 2010 Optimized Tersoff and Brenner empirical potential parameters for lattice dynamics and phonon thermal transport in carbon nanotubes and graphene. Phys. Rev. B 81, 205441. (doi:10.1103/PhysRevB.81.205441)

70. Mortazavi B, Ahzi S. 2013 Thermal conductivity and tensile response of defective graphene: a molecular dynamics study. Carbon 63, 460-470. (doi:10.1016/j.carbon.2013.07.017)

71. Zang JL, Zhao YP. 2012 Silicon nanowire reinforced by single-walled carbon nanotube and its applications to anti-pulverization electrode in lithium ion battery. Compos. B Eng. 43, 76-82. (doi:10.1016/j.compositesb.2011.04.034)

72. Chandra A, Krishnan N, Patra PK, Ghosh D. 2017 Coaxial boron-nitride/carbon nanotubes as a potential replacement for double-walled carbon nanotubes for high strain applications. J. Nanosci. Nanotechnol. 17, 5252-5260. (doi:10.1166/jnn.2017.13819)

73. Sircar A, Patra PK. 2019 A simple generalization of Prandtl-Tomlinson model to study nanoscale rolling friction. (http://arxiv.org/abs/1912.00232).

74. Martyna GJ, Klein ML, Tuckerman M. 1992 Nosé-Hoover chains: the canonical ensemble via continuous dynamics. J. Chem. Phys. 97, 2635-2643. (doi:10.1063/1.463940)

75. Patra P, Bhattacharya B. 2014 A deterministic thermostat for controlling temperature using all degrees of freedom. J. Chem. Phys. 140, 064106. (doi:10.1063/1.4864204)

76. Hoover WG, Sprott JC, Patra PK. 2015 Ergodic time-reversible chaos for Gibbs' canonical oscillator. Phys. Lett. A 379, 2935-2940. (doi:10.1016/j.physleta.2015.08.034)

77. Patra PK, Sprott JC, Hoover WG, Hoover CG. 2015 Deterministic time-reversible thermostats: chaos, ergodicity, and the zeroth law of thermodynamics. Mol. Phys. 113, 2863-2872. (doi:10.1080/00268976.2015.1026856)

78. Soroush R, Koochi A, Kazemi A, Noghrehabadi A, Haddadpour H, Abadyan M. 2010 Investigating the effect of Casimir and van der Waals attractions on the electrostatic pull-in instability of nano-actuators. Phys. Scr. 82, 045801. (doi:10.1088/0031-8949/82/04/045801)

79. Batra R, Porfiri M, Spinello D. 2007 Effects of Casimir force on pull-in instability in micromembranes. EPL (Europhys. Lett.) 77, 20010. (doi:10.1209/0295-5075/77/20010) 